\title{
Synthesis of multilamellar walls vesicles $(M L W V)$ polyelectrolyte surfactant complexes (PESCs) from pH-stimulated phase transition using microbial biosurfactants
}

\section{Chloé Seyrig ${ }^{a}$, Patrick Le Griel ${ }^{\mathrm{a}}$, N. Cowieson, ${ }^{\mathrm{b}}$ J. Perez, ${ }^{\mathrm{c}}$ Niki Baccile $^{\mathrm{a}}$}

a Sorbonne Université, Centre National de la Recherche Scientifique, Laboratoire de Chimie de la Matière Condensée de Paris , LCMCP, F-75005 Paris, France

${ }^{\mathrm{b}}$ Diamond Light Source Ltd, Diamond House, Harwell Science \& Innovation Campus, Didcot, Oxfordshire, OX11 0DE

c Synchrotron SOLEIL, L'Orme des Merisiers Saint-Aubin, BP 4891192 Gif-sur-Yvette Cedex

\begin{abstract}
Multilamellar wall vesicles $(M L W V)$ are an interest class of polyelectrolyte-surfactant complexes (PESCs) for the wide applications ranging from house-care to biomedical products. If $M L W V$ are generally obtained by a polyelectrolyte-driven vesicle agglutination under pseudoequilibrium conditions, the resulting phase is often a mixture of more than one structure. In this work, we show that $M L W V$ can be massively and reproductively prepared from a recentlydeveloped method involving a $\mathrm{pH}$-stimulated phase transition from a complex coacervate phase (Co). We employ a biobased $\mathrm{pH}$-sensitive microbial glucolipid biosurfactant in the presence of a natural, or synthetic, polyamine (chitosan, poly-L-Lysine, polyethylene imine, polyallylamine). In situ small angle X-ray scattering (SAXS) and cryogenic transmission electron microscopy (cryo-TEM) show a systematic isostructural and isodimensional transition from the $C o$ to the $M L W V$ phase, while optical microscopy under polarized light experiments and cryo-TEM reveal a massive, virtually quantitative, presence of $M L W V$. Finally, the multilamellar wall structure is not perturbed by filtration and sonication, two typical methods employed to control size distribution in vesicles. In summary, this work highlights a new, robust, non-equilibrium phase-change method to develop biobased multilamellar wall vesicles, promising soft colloids with applications in the field of personal care, cosmetics and pharmaceutics among many others.
\end{abstract}

Keywords. Polyelectrolyte-Surfactant Complex (PESC), complex coacervates, biosurfactants, polyelectrolytes, multilamellar walls vesicles 


\section{Introduction}

Polyelectrolytes and surfactants may assemble into complex structures known as polyelectrolyte-surfactant complexes (PESCs). When these compounds are oppositely charged, their self-assembly process is mainly driven by electrostatic interactions and it results in the formation of aggregates, which have a broad range of applications in biological materials, ${ }^{1-5}$ drug delivery, ${ }^{6-8}$ surface modifications, ${ }^{9}$ colloid stabilization ${ }^{10}$ and flocculation ${ }^{11}$ and consumer health-care products. The rich mesoscopical and structural organisation of surfactants combined with the electrostatic interactions with polyelectrolytes give rise to a wide range of structures and phases. ${ }^{12-17}$ Many works reported cubic or hexagonal mesophases ${ }^{15,16}$ but also a number of micellar-based structures: pearl-necklace morphologies, ${ }^{2,18,19}$ interpenetrated polyelectrolytewormlike/cylindrical micelles network, ${ }^{18,20-22}$ spheroidal clusters composed of densely packed micelles held by the polyelectrolyte, the latter known as complex coacervates when they form a liquid-liquid phase separation. ${ }^{18,23,24}$

Very interesting PESCs structures are formed when the surfactant forms low curvature vesicular morphologies. It is in fact generally admitted that modifying vesicles by the addition of polyelectrolytes is an interesting, cheap and simple approach to obtain nanocapsules, ${ }^{22}$ which are good candidates to be used as versatile delivery systems, ${ }^{18,22}$ like gene delivery, ${ }^{1,21,25,26}$ or as MRI contrast agents. ${ }^{27}$ One of the first PESCs vesicular systems has been reported more than 20 years ago in DNA-CTAB (cetytrimethylammonium bromide) systems, which were the precursors of a number of carriers for gene transfection and often referred to as lipoplexes, when cationic lipids replace surfactants in DNA complexation. ${ }^{28,29}$ If the term lipoplexe supposes the use of nucleic acids as complexing agents, similar structures, often addressed to as onion-like structures ${ }^{30}$ or multilamellar vesicles, ${ }^{13}$ were observed using both lipids and surfactants complexed by a wide range of polyelectrolytes. However, multilamellar, or onionlike, vesicles are rather characterized by single-wall membranes concentrically distributed from the outer to the inner core of the vesicle. Lipoplexes, on the contrary, are vesicular objects with a large lumen and a dense multilamellar wall. For this reason, in this work we employ the name multilamellar wall vesicles $(M L W V)$.

The mechanism of formation of $M L W V$ was addressed by several authors, but a common agreement is not achieved, yet. Several works propose that the lipid:polyelectrolyte ratio controls the fusion of single-wall vesicles $M L W V,{ }^{18,28,31-34}$ while others rather observe vesicular agglutination under similar conditions. ${ }^{35-37}$ In fact, a general consensus has not been found and a multiphasic system including agglutinated vesicles and $M L W V$ are actually observed. ${ }^{38}$ The question whether or not $M L W V$, and PESCs in general, are equilibrium structures and how they 
are formed is still open, especially when they are prepared under non-equilibrium conditions. ${ }^{18}$ To the best of our knowledge, the only works exploring a stimuli-induced approach in the synthesis of MLWV in particular, and PESCs in general, were proposed by Chiappisi et al.. ${ }^{20,39}$ However, the $\mathrm{pH}$ variation in these work was still performed under pseudo-equilibrium conditions with equilibration times ranging from 2 to 15 days for each $\mathrm{pH}$ value.

In a recent work, we have explored a Co-to- $M L W V$ phase transition under nonequilibrium conditions using a continuous variation in $\mathrm{pH},{ }^{40}$ as illustrated by Figure 1 . We could show that in the presence of G-C18:1, an acidic microbial glycolipid biosurfactant, ${ }^{41,42}$ and poly-L-lysine (PLL), a cationic polyelectrolyte (PEC), the pH-stimulated micelle-to-vesicle phase transition of the lipid drives a continuous, isostructural and isodimensional, transition between complex coacervates and multilamellar wall vesicles. In the present work, we generalize the method of preparing $M L W V$ through a phase transition approach performed under non-equilibrium conditions and we show its performance in comparison to the more accepted method of vesicular agglutination. We show that this method can be applied to a broader set of polyelectrolytes and we explore in more detail the structure and size control of $M L W V$.

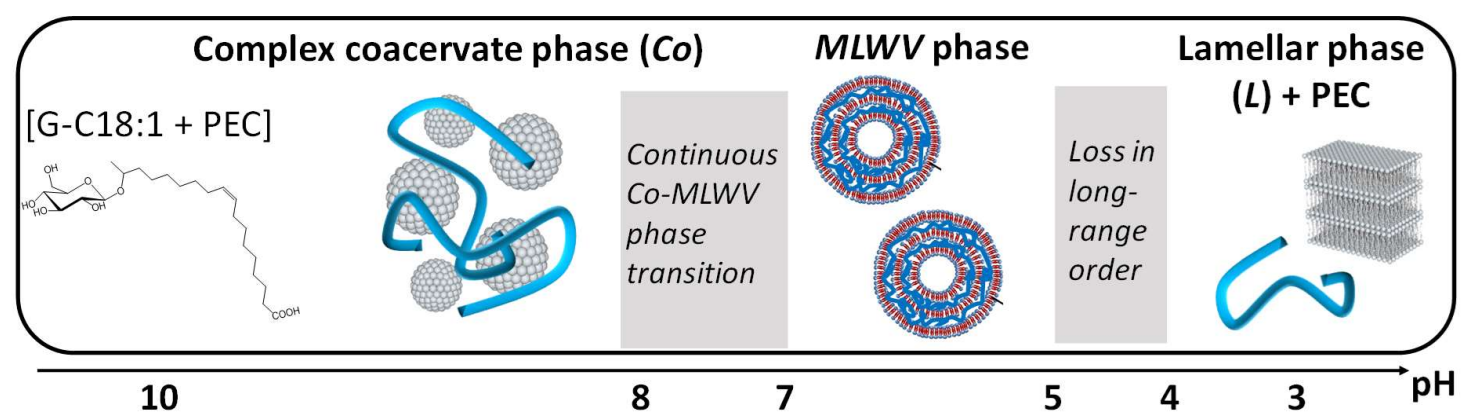

Figure 1 - Phase transition and structures obtained by mixing G-C18:1 and PEC upon a rapid variation of pH. Starting from pH 11, G-C18:1 micelles and free polymer chains coexist until pH $\sim 9$ where they interact to form complex coacervates $(\mathrm{Co})$. They progressively rearrange into multilamellar wall vesicles $(M L W V)$ and dissociate below $\mathrm{pH} \sim 4$, where G-C18:1 forms a lamellar phase coexisting with free polymer chains.

\section{Experimental section}

\section{Chemicals}

In this work we use microbial glycolipids G-C18:1, made of a single $\beta$-D-glucose hydrophilic headgroup and a C18 fatty acid tail (monounsaturation in position 9,10). From alkaline to acidic $\mathrm{pH}$, the former undergoes a micelle-to-vesicle phase transition. ${ }^{41}$ The syntheses of glucolipid G-C18:1 is described in $\operatorname{Ref}^{43}$ and ${ }^{42}$, where the typical ${ }^{1} \mathrm{H}$ NMR spectra and HPLC chromatograms are given. The compound used in this work have a molecular purity of more than $95 \%$. 
The polyelectrolytes used in this work are chitosan, obtained from the deacetylation of chitin from crusteans' shells, poly-L-lysine, widely used in biomedical field, and polyethylenimine. Chitosan oligosaccharide lactate (CHL) $\left(M_{\mathrm{w}} \approx 5 \mathrm{KDa}, \mathrm{p} K_{\mathrm{a}} \sim 6.5\right)^{44}$ with a deacetylation degree $>90 \%$, poly-L-lysine (PLL) hydrobromide $\left(M_{\mathrm{w}} \approx 1-5 \mathrm{KDa}, \mathrm{p} K_{\mathrm{a}} \sim 10\right.$ 10.5), ${ }^{45}$ polyallyllamine hydrochloride $(\mathrm{PAH})\left(M_{\mathrm{w}} \approx 1-5 \mathrm{KDa}, \mathrm{p} K_{\mathrm{a}} \sim 9.5\right),{ }^{45}$ polyethylenimine (PEI) hydrochloride (linear, $\left.M_{\mathrm{w}} \approx 4 \mathrm{KDa}, \mathrm{p} K_{\mathrm{a}} \sim 8\right)^{46}$ and gelatin (type A, from porcine skin, $M_{\mathrm{w}}$ $\approx 50-100 \mathrm{KDa}$, isoelectric point 7-9) are purchased from Sigma-Aldrich. All other chemicals are of reagent grade and are used without further purification.

\section{Preparation of stock solutions}

G-C18:1 $\left(C=5 \mathrm{mg} \cdot \mathrm{mL}^{-1}, C=20 \mathrm{mg} \cdot \mathrm{mL}^{-1}\right), \operatorname{CHL}\left(C=2 \mathrm{mg} \cdot \mathrm{mL}^{-1}\right), \operatorname{PLL}\left(C=5 \mathrm{mg} \cdot \mathrm{mL}^{-1}\right.$, $\left.C=20 \mathrm{mg} \cdot \mathrm{mL}^{-1}\right)$, PEI $\left(C=5 \mathrm{mg} \cdot \mathrm{mL}^{-1}\right)$, PAH $\left(C=2 \mathrm{mg} \cdot \mathrm{mL}^{-1}\right)$ and gelatin $\left(C=5 \mathrm{mg} \cdot \mathrm{mL}^{-1}\right)$ stock solutions are prepared by dispersing the appropriate amount of each compound in the corresponding amount of Milli-Q-grade water. The solutions are stirred at room temperature ( $\left.T=23 \pm 2{ }^{\circ} \mathrm{C}\right)$ and the final $\mathrm{pH}$ is increased to 11 by adding a few $\mu \mathrm{L}$ of $\mathrm{NaOH}(C=0.5 \mathrm{M}$ or $C=1 \mathrm{M})$.

\section{Preparation of samples}

Samples are prepared by mixing appropriate volume ratios of G-C18:1 stock solutions at pH 11 and cationic polyelectrolyte (PEC) stock solutions, as defined in Table 1. The final total volume is generally set to $V=1 \mathrm{~mL}$ or $V=2 \mathrm{~mL}$, the solution $\mathrm{pH}$ is about 11 and the final concentrations are given in Table 1. The $\mathrm{pH}$ of the mixed lipid-PEC solution is eventually decreased by the addition of $1-10 \mu \mathrm{L}$ of a $\mathrm{HCl}$ solution at $C=0.5 \mathrm{M}$ or $C=1 \mathrm{M}$. The rate at which $\mathrm{pH}$ is changed is generally not controlled although it is in the order of several $\mu \mathrm{L} \cdot \mathrm{min}^{-1}$. Differently than in other systems, ${ }^{47,48}$ we did not observe unexpected effects on the PESC structure to justify a tight control over the $\mathrm{pH}$ change rate. 
Table 1 - Relative volumes of G-C18:1 and cationic polyelectrolyte (PEC) solutions to mix to obtain given concentrations

\begin{tabular}{|c|c|c|c|c|}
\hline \multicolumn{3}{|c|}{ Volume } & \multicolumn{2}{c|}{ Concentration } \\
\hline $\begin{array}{c}\text { G-C18:1 stock } \\
\text { solution } / \mathrm{mL}\end{array}$ & $\begin{array}{c}\text { PEC stock } \\
\text { solution } / \mathrm{mL}\end{array}$ & Water $/ \mathrm{mL}$ & $\begin{array}{l}C_{\mathrm{G}-\mathrm{C} 18: 1} / \\
\mathrm{mg} \cdot \mathrm{mL}^{-1}\end{array}$ & $C_{\mathrm{PEC}} /{\mathrm{mg} \cdot \mathrm{mL}^{-1}}^{-1}$ \\
\hline \multirow{3}{*}{0.5} & 0.5 & 0 & 2.5 or 10 & 2.5 or 10 \\
\cline { 2 - 5 } & 0.25 & 0.25 & 2.5 & 1.25 \\
\cline { 2 - 5 } & 0.125 & 0.375 & 2.5 & 0.625 \\
\hline
\end{tabular}

\section{pH-resolved in situ Small angle X-ray scattering (SAXS)}

In situ SAXS experiments during $\mathrm{pH}$ variation are performed at room temperature on two different beamlines. The B21 beamline at Diamond Light Source Synchrotron (Harwell, England) is employed using an energy of $E=13.1 \mathrm{keV}$ and a fixed sample-to-detector (Eiger 4M) distance of $2.69 \mathrm{~m}$. The Swing beamline at Soleil Synchrotron (Saint-Aubin, France) is employed using an energy of $E=12 \mathrm{keV}$ and a fixed sample-to-detector (Eiger X 4M) distance of $1.995 \mathrm{~m}$. For all experiments: the $q$-range is calibrated to be contained between $\sim 5 \cdot 10^{-3}<$ $q / \AA^{-1}<\sim 4.5 \cdot 10^{-1}$; raw data collected on the $2 \mathrm{D}$ detector are integrated azimuthally using the inhouse software provided at the beamline and so to obtain the typical scattered intensity $I(q)$

profile, with $q$ being the wavevector $(q=4 \pi \sin \theta / \lambda$, where $2 \theta$ is the scattering angle and $\lambda$ is the wavelength). Defectuous pixels and beam stop shadow are systematically masked before azimuthal integration. Absolute intensity units are determined by measuring the scattering signal of water $\left(I_{q=0}=0.0163 \mathrm{~cm}^{-1}\right)$.

The same sample experimental setup is employed on both beamlines: the sample solution $(V=1 \mathrm{~mL})$ with the lipid and PEC at their final concentration and $\mathrm{pH} \sim 11$ is contained in an external beaker under stirring. The solution is continuously flushed through a $1 \mathrm{~mm}$ glass capillary using an external peristaltic pump. The $\mathrm{pH}$ of the solution in the beaker is changed using an interfaced push syringe, injecting microliter amounts of a $0.5 \mathrm{M} \mathrm{HCl}$ solution. $\mathrm{pH}$ is measured using a micro electrode (Mettler-Toledo) and the value of $\mathrm{pH}$ is monitored live and manually recorded from the control room via a network camera pointing at the $\mathrm{pH}$-meter located next to the beaker in the experimental hutch. Considering the fast $\mathrm{pH}$ change kinetics, the error on the $\mathrm{pH}$ value is \pm 0.5 . 


\section{Polarized Light Microscopy (PLM)}

PLM experiments are performed with a transmission Zeiss AxioImager A2 POL optical microscope. A drop of the given sample solution is deposited on a slide covered with a cover slip. The microscope is equipped with a polarized light source, crossed polarizers and an AxioCam CCD camera.

\section{Cryogenic transmission electron microscopy (cryo-TEM)}

Cryo-TEM experiments are carried out on an FEI Tecnai 120 twin microscope operated at $120 \mathrm{kV}$ and equipped with a Gatan Orius CCD numeric camera. The sample holder is a Gatan Cryoholder (Gatan 626DH, Gatan). Digital Micrograph software is used for image acquisition. Cryofixation is done using a homemade cryofixation device. The solutions are deposited on a glow-discharged holey carbon coated TEM copper grid (Quantifoil R2/2, Germany). Excess solution is removed and the grid is immediately plunged into liquid ethane at $-180^{\circ} \mathrm{C}$ before transferring them into liquid nitrogen. All grids are kept at liquid nitrogen temperature throughout all experimentation. Images were analyzed using Fiji software, available free of charge at the developer's website. ${ }^{49}$

\section{Results}

In recent publications, ${ }^{40,50}$ we have explored the complex coacervation between microbial glycolipids and cationic polyelectrolytes (PEC). For this reason, this aspect is only briefly shown in here. Cryo-TEM images presented in Figure 2 show the structure of PECcomplexed G-C18:1 complex coacervates above $\mathrm{pH}$ 7. Irrespective of the selected PEC, all systems show spheroidal colloids of variable size in the $100 \mathrm{~nm}$ range. One can identify two types of structures, both typical of complex coacervates: ${ }^{23,24,50,51}$ dense aggregated structures, shown in Figure 2a,c and very similar to what was found by us ${ }^{40,50}$ and others, ${ }^{23}$ are attributed to dehydrated, densely-packed, micelles tightly interacting with the polyelectrolyte; a biphasic medium composed of spheroidal, poorly-contrasted, colloids embedded in a textured medium describe hydrated structures of less defined composition, probably describing an intermediate of coacervation step. The latter were also reported by us ${ }^{40,50}$ and others. ${ }^{51,52}$ In all cases, the complex coacervate phase $(\mathrm{Co})$ is a PESC forming in the micellar region of the surfactant's phase diagram and having the specificity of a liquid-liquid phase separation, ${ }^{18,24}$ compared to other supramicellar PESCs undergoing a solid-liquid phase separation. ${ }^{18}$ 
a)
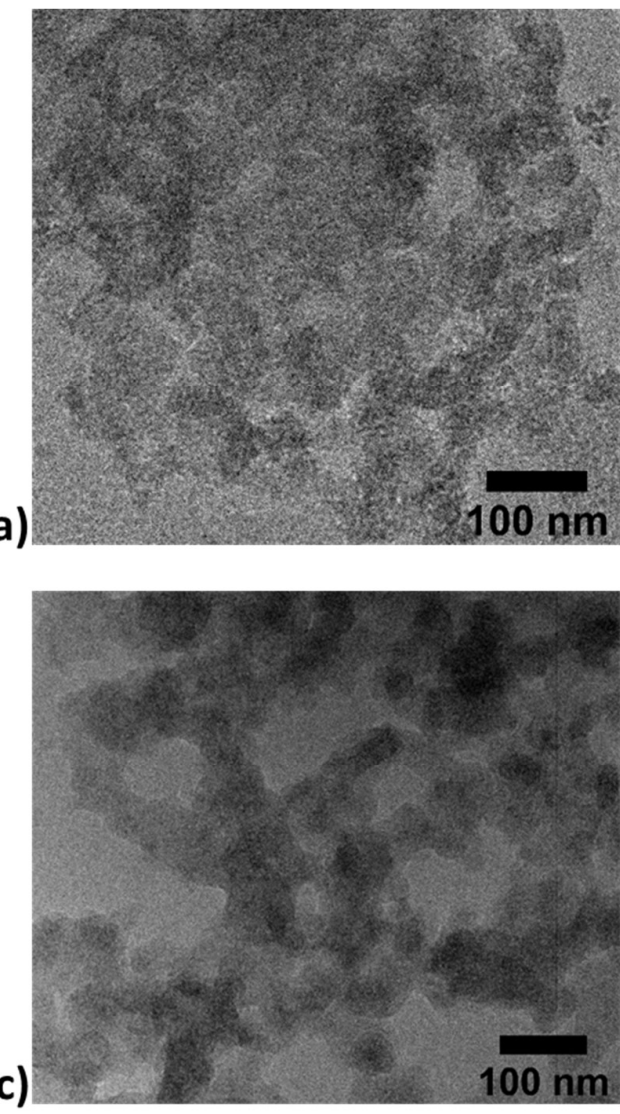

b)

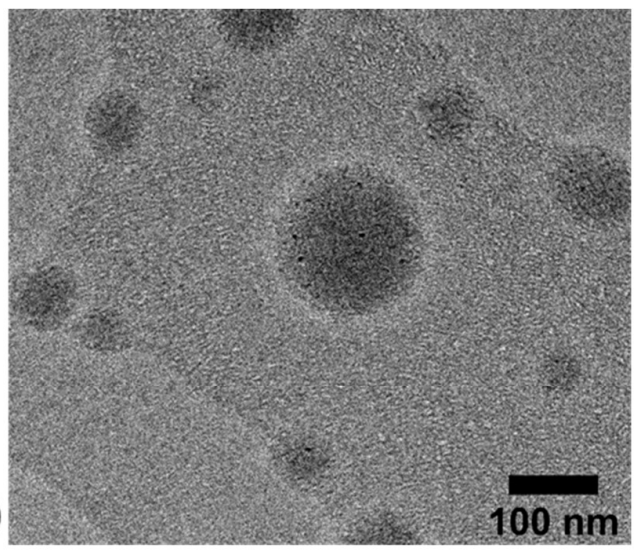

d)

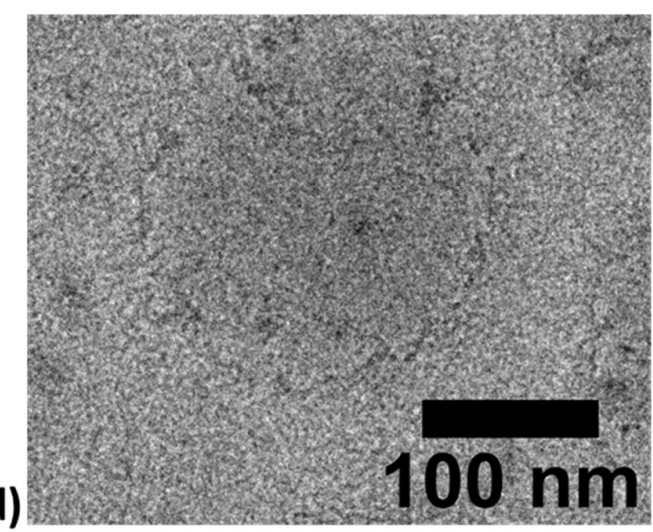

Figure 2 - Cryo-TEM images of PESC solutions in the complex coacervate phase composed of G-C18:1 lipid complexed with a) CHL (pH 7.16), b) PLL (pH 9.16), c) PAH (pH 8.96) and d) PEI (pH 9.02). $C_{\mathrm{G}-\mathrm{Cl} 18: 1}=C_{\mathrm{PEI}}=$ $2.5 \mathrm{mg} \cdot \mathrm{mL}^{-1}, C_{\mathrm{CHL}}=1 \mathrm{mg} \cdot \mathrm{mL}^{-1}, C_{\mathrm{PAH}}=0.25 \mathrm{mg} \cdot \mathrm{mL}^{-1}, C_{\mathrm{PLL}}=1.25 \mathrm{mg} \cdot \mathrm{mL}^{-1}$

The difference between dense and poorly-contrasted structures is PEC-independent and it is more related to the stage of coacervation. At an early stage, colloids with a relatively low electron density form and coexist with a rich micellar phase. Free micelles progressively interact with residual polymer chains. At a later, entropy-driven (dehydration and counterion release), ${ }^{53}$ stage of coacervation, droplets with a higher electron density massively form. Unfortunately, neither the texture of the particles nor their internal structure can be easily controlled as they strongly depend on the type of PEC, its stiffness, charge density, stage of coacervation and even kinetics. For these reasons, isolating a specific structure in a Co phase can be challenging and we have ourselves found coexisting dense and poorly-contrasted structures, ${ }^{40}$ thus preventing any reasonable structure-composition generalization concerning the images presented in Figure 2. 

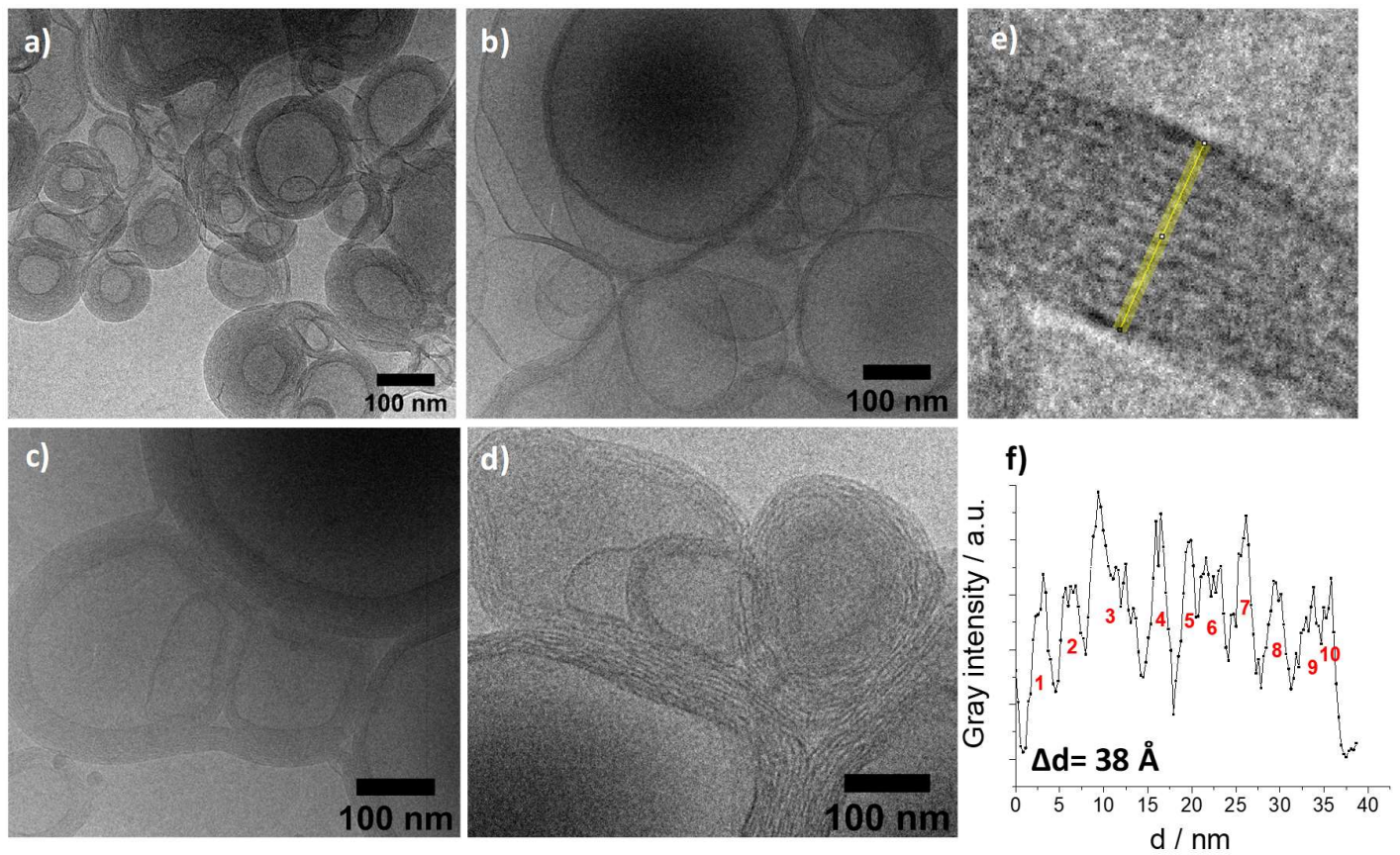

Figure 3 - Cryo-TEM images of a $M L W V$ phase composed of acidic G-C18:1 lipid complexed with a) CHL (pH 4.87), b) PLL (pH 4.70), c) PAH (pH 4.25) and d) PEI (pH 5.33). $C_{\mathrm{G}-\mathrm{C} 18: 1}=C_{\mathrm{PEI}}=C_{\mathrm{PLL}}=2.5 \mathrm{mg} \mathrm{mL}^{-1}$, $C_{\mathrm{CHL}}=1 \mathrm{mg} \cdot \mathrm{mL}^{-1}, C_{\mathrm{PAH}}=0.25 \mathrm{mg} \cdot \mathrm{mL}^{-1}$. e) Zoomed cryo-TEM image of [G-C18:1 + PLL] mixture and its corresponding profile (f) allowing the determination of the interlamellar distance. Cryo-TEM data have been analyzed using Fiji software. ${ }^{49}$

At $\mathrm{pH}$ below 7, vesicular structures with multilamellar walls ( $M L W V$ phase) are observed by cryo-TEM for all PEC samples (Figure 3). These structures are closely-related to a lipoplexe-type phase rather than to an onion-like phase, whereas the latter is composed of concentric single-wall vesicles, while the former keeps a free lumen and a thick multilamellar wall. ${ }^{28}$ Figure 3 also shows a strong packing of the multilamellar walls as well as a strong interconnection between adjacent vesicular objects, in agreement with lipoplexes and other multilamellar wall vesicles reported in the literature. ${ }^{22}$ The walls are constituted of alternating sandwiched layers composed of tightly packed polyelectrolyte chains and interdigitated layers of G-C18:1. ${ }^{40} d$-spacing can be directly estimated from cryo-TEM images (Figure 3e,f) and we find a set of values of $d=33.7 \pm 4.95 \AA$ for the PLL system and $d=31.6 \pm 3.00 \AA, 25.3 \pm 4.60$ $\AA$ and $41.1 \pm 0.30 \AA$ respectively for CHL, PAH and PEI systems. Within the error, these values are compatible with interdigitated G-C18:1 layers, ${ }^{40-42}$ of which the thickness can be estimated to be around $30 \AA$ by applying the Tanford relationship, ${ }^{54}$ but also close to what is classically recorded for lipoplexes. ${ }^{21,22,32}$ One should note an interesting feature on Figure $3 \mathrm{~d}$ : the multilamellar walls of the PECSs involving PEI appear less tightly packed and more disordered 
than for other PESCs. This effect may be a consequence of the freezing protocol, although all samples have been frozen in the same way, or related to the specific use of PEI. Another hypothesis, which does not exclude the previous one, could be that the local disorder results from electrostatically induced undulations of the membrane, as already reported on lamellar DNA-lipid complexes. ${ }^{55}$

Cryo-TEM images recorded on the Co (Figure 2) and $M L W V$ (Figure 3) phases show that the $C o-$ to- $M L W V$ transition is a general property of G-C18:1 PESCs: it strictly depends on the lipid phase behavior, while the polyelectrolyte only guarantees the cohesion between the lipid membranes. We highlighted elsewhere ${ }^{40}$ by $\mathrm{pH}$-resolved in situ SAXS experiments an explicit isostructural and isodimensional continuity in the Co-to- $M L W V$ phase transition: the broad correlation peak at $q=0.171 \AA^{-1}$ ( $d$-spacing of $36.7 \AA$ ) of the coacervate phase coexists with the sharp diffraction peak of the $M L W V$ phase at $q_{1}=0.178 \AA^{-1}(d$-spacing of $35.3 \AA$ ) in a narrow range around $\mathrm{pH} 7 .{ }^{40}$ Restructuring is driven by the progressive hydrogenation of the carboxylates group and the resulting conformational change of the lipid, which favors the formation of low curvature colloids, while inter-lipid repulsive electrostatic interactions disappear in the meanwhile.

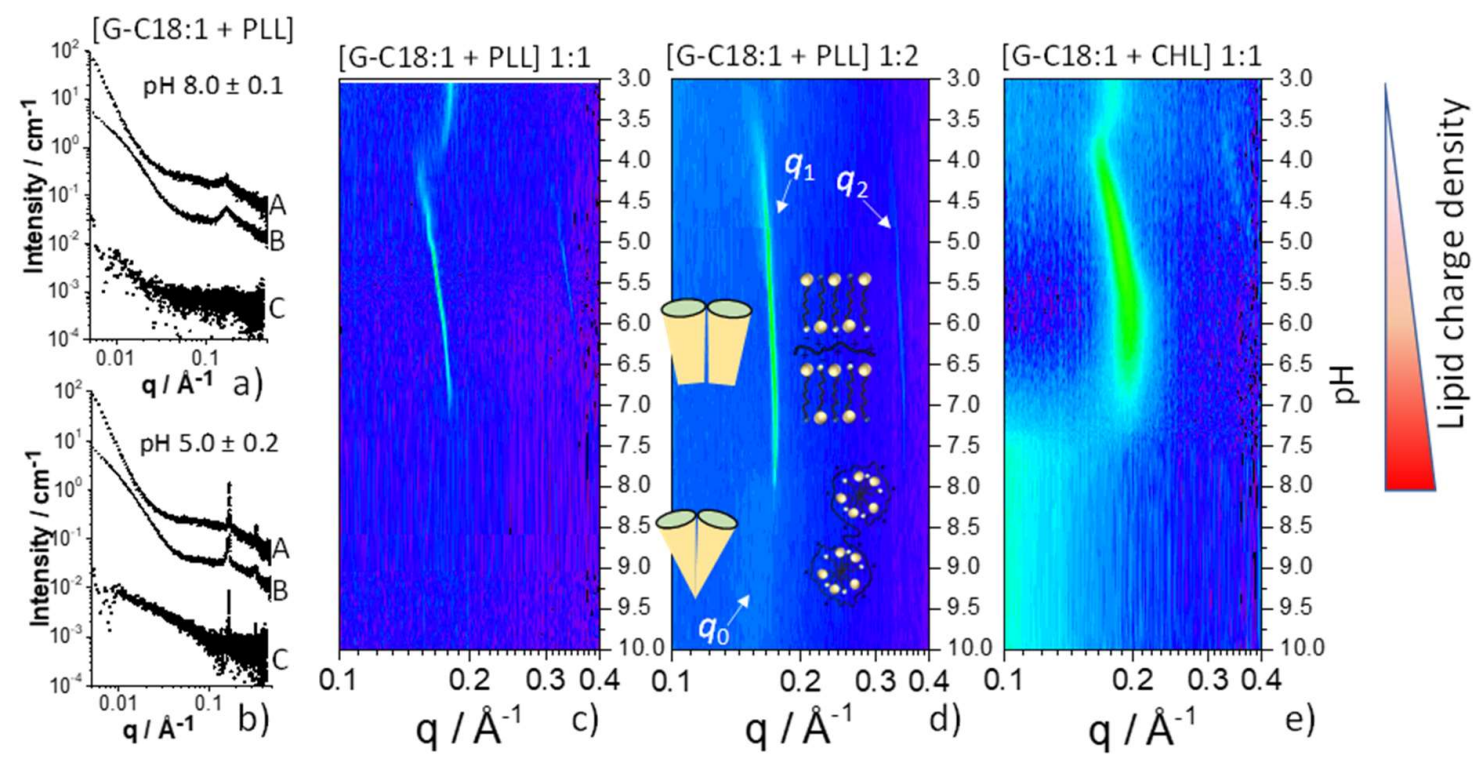

Figure 4 - SAXS profiles of [G-C18:1 + PLL] PESCs at a) basic and b) acidic pH with G-C18:1:PLL concentration ratios in $\mathrm{mg}^{-1} \mathrm{~mL}^{-1}: A=2.5: 5, B=10: 10, C=2.5: 2.5$. c-e) 2D SAXS contour plots of G-

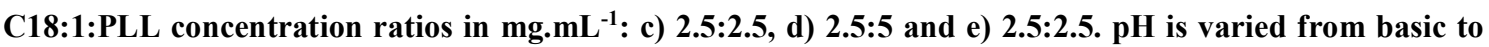
acidic. 
SAXS profiles presented Figure 4Figure 4 show two different behaviors of the mixture [G-C18:1 + PLL]: at basic pH (Figure 4a), a broad correlation peak is observed at about $q=0.17$ $\AA^{-1}$ for all lipid:PLL ratios, where the peak can be more pronounced either with concentration (B profile) or lipid:PLL ratio (A profile). SAXS profiles B and C were previously assigned to complex coacervates, and more details on the structure of the Co phase can be found in Ref. ${ }^{40}$. In similar systems, the slope at low $q$ was shown to be indicative of the shape of the PESC; 39 here, the slope is below -3 . If such values are typical of fractal interfaces, ${ }^{56,57}$ we cannot unfortunately draw any conclusion on the structure of the complex coacervates, most likely because the Co phase in these systems is heterogeneous. ${ }^{40}$

Below pH 7 (Figure 4b), a sharp diffraction peak and its first harmonics are visible respectively around $q_{1}=0.17 \AA^{-1}$ and $q_{2}=0.34 \AA^{-1}$, characteristic of the (100) and (200) reflections of a lamellar order in the walls, described previously and shown in Figure 3. The $d$ spacing of $37 \AA$ is in agreement with the ones deduced from cryo-TEM (Figure 3e,f). Similar results are obtained at different lipid:PLL ratios (Figure 4c,d) but also for other PEC. Figure $S$ 1 presents the SAXS signals of [G-C18:1 + CHL] solutions at basic and acidic $\mathrm{pH}$, compared to the control solutions of [G-C18:1] and [CHL] alone as well as their arithmetic sum. If at acidic $\mathrm{pH}$ the signature of the lamellar wall of the mixture compared to the controls is out of doubt, the signal at basic $\mathrm{pH}$ is less straightforward to interpret, due to the scattering of $\mathrm{CHL}$ alone, known to precipitate above $\mathrm{pH} 7 .^{58}$ This result is similar to what was found for acidic deacetylated sophorolipids; ${ }^{40,50}$ however, considering the fact that cryo-TEM experiments suggest the presence of complex coacervates, we cannot exclude their formation, although their content may constitute a small minority, if compared to the PLL-based PESCs in the same $\mathrm{pH}$ range. Another argument for the formation of $\mathrm{Co}$ in the presence of CHL will be given later.

Figure 4c-e show the pH-resolved in situ contour plots of [G-C18:1 + PLL] PESCs at various lipid:PLL ratio and with CHL. They are recorded between $\mathrm{pH} 10$ and 3 and focus on the high- $q$ region of the SAXS pattern, sensitive to the structural Co-to- $M L W V$ phase transition. All pH-resolved in situ contour plots in Figure 4 show three common features: 1) the Co-to$M L W V$ transition between $\mathrm{pH} 8$ and 7 , where $q_{1}$ and $q_{2}$ refer to the first and second order peaks of the lamellar wall; 2) a low- $q$ shift of $q_{1}$ and $q_{2}$ when $\mathrm{pH}$ decreases to about 4.5 , indicating a swelling of the lamellar period, and 3) a loss of the signal between about $\mathrm{pH} 4.5$ and $\mathrm{pH} 3.5$, below which a constant peak at higher $q$-values (generally around $q=0.2 \AA^{-1}$ ) appears. These phenomena were quantitatively described in more detail in Ref. ${ }^{40}$ and will only be summarized hereafter. 
When fully deprotonated at basic $\mathrm{pH}, \mathrm{G}-\mathrm{C} 18: 1$ is in a high curvature, micellar, environment (Co phase) at basic $\mathrm{pH}$. This state, represented by the drawing superimposed on Figure $4 \mathrm{~d}$, is proven by both cryo-TEM and the broad correlation peak at about $q_{0}=0.17 \AA^{-1}$. After crossing the transition $\mathrm{pH}$ range between 8 and 7, the number of negative charges decreases and G-C18:1 is entrapped in a low-curvature, interdigitated layer, environment. The continuity between $q_{0}$ and $q_{1}$ strongly suggest an isostructural and isodimensional transition between the micelle and membrane configutations, without any loss of the interaction with the polyelectrolyte. This is also sketched on Figure $4 \mathrm{~d}$. When the $\mathrm{pH}$ is decreased further, the $\mathrm{COOH}$ content increases and thus the membrane charge density decreases. The interlamellar distance consequently increases due to the repulsive pressure exerted by the charged polyelectrolyte, which undergoes hydration and increase internal electrostatic repulsion. . $^{2,59,60}$ When hydrogenation of carboxylate groups reach a certain extent, attractive interaction with PLL can no longer hold the membranes together and $M L W V$ then lose their long-range lamellar order, which results in their complete disruption and the concomitant expulsion of PLL. Below $\mathrm{pH} 3$, this mechanism leads to the precipitation of a polyelectrolyte-free lamellar, $L$, phase, which is also observed for PEC-free G-C18:1 solutions.

A closer look at the experiments in Figure 3 indicates two additional features. The $\mathrm{pH}$ stability domain of the $M L W V$ phase seems to vary with the lipid:PLL ratio. Comparison of Figure $3 \mathrm{c}$ and Figure 3d, respectively recorded at lipid:PLL $=1: 1$ and 1:2 reveal that the $q_{1}$ peak of the $M L W V$ phase is observed between $\mathrm{pH} 8$ and 7. At the 1:2 ratio the $M L W V$ phase starts at about $\mathrm{pH} 8$ while at the $1: 1$ ratio the $M L W V$ phase is only visible at $\mathrm{pH}$ is below 7 . At higher concentrations $\left(C=10 \mathrm{mg} \cdot \mathrm{mL}^{-1}\right)$, but still for a 1:1 ratio, the stability frontier seems to be shifted at $\mathrm{pH}$ of about 7.5. ${ }^{40}$ Although we do not have enough data to draw a general trend, it is wellknown that the lipid:polyelectrolyte ratio reflects the negative:positive charge ratio and for this reason it has a direct impact on the electroneutrality, thus affecting a number of structural features of PESCs: the wall thickness of the multilamellar structure, ${ }^{20,61}$ the PESC morphology and colloidal stability. ${ }^{18}$ For instance, order is noticeably improved when the charge ratio approaches 1:1, ${ }^{62}$ and micelle-polyelectrolyte complex coacervation can be favoured or not. ${ }^{63}$ This ratio is particularly crucial to control the properties of the lipoplexes and thus their applications: lipid/DNA ratio was reported to influence both the formation of lipoplexes and the release of $\mathrm{DNA}^{64}$ and gene transfer activity. ${ }^{65}$ Many authors have shown that the lipid:polyelectrolyte ratio actually controls the formation of $M L W V$ structures ${ }^{18,28,31-34}$ over agglutinated single-wall vesicles, ${ }^{35-37}$ but in fact it is more likely that a general consensus has not been found, yet, and reality often consists in a mixtures of $M L W V$ and agglutinated 
vesicles, ${ }^{38}$ although many authors do not specify it. One of the reasons that could explain such discrepancy is the parallel influence of several other parameters like the charge density on both the lipid membrane and in the polyelectrolytes, the rigidity of the latter, the bending energy of the lipid membrane, the ionic strength and so on. ${ }^{14,18}$ In the present case, it is important to note that: 1) G-C18:1 forms a stable $M L W V$ phase with all PEC tested in this work and of different origin (biobased vs. synthetic) and rigidity. 2) Multilamellar wall vesicles are stable in the neutral $\mathrm{pH}$ range, which can be a good opportunity for applications in the biomedical field, for instance.

An interesting remark concerns the long-range order inside the vesicular multilamellar walls. The width of the lamellar peak around $q \sim 0.2 \AA^{-1}$ is more than ten times larger for the CHL (Figure 4e, $\Delta q \sim 3.10^{-2} \AA^{-1}$ ) than the PLL (Figure 4c,d, $\Delta q \sim 2.10^{-3} \AA^{-1}$ ) system, either suggesting an average smaller size of the lamellar domains or a poorer lamellar order in the case of the $M L W V$ obtained from CHL. The reason behind such difference could be the bulkiness and stiffness of CHL with respect to PLL, ${ }^{31}$ but one should recall from Figure 2 and related discussion that [G-C18:1 + CHL] solutions do not form an extensive Co phase. We have already made the hypothesis that the Co phase is necessary to form the $M L W V$ phase, ${ }^{40}$ and we will reinforce this assumption in the next part of this work.

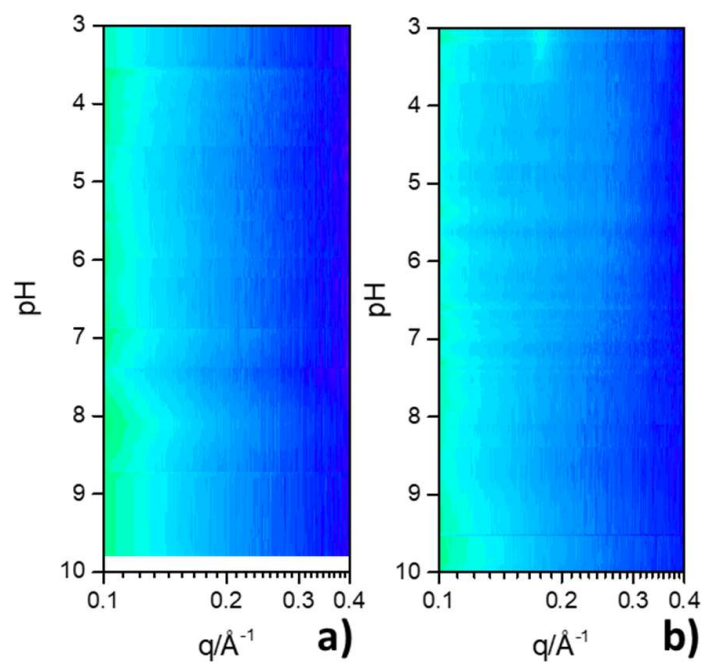

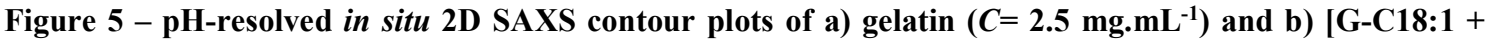
gelatin] mixture $\left(C_{\mathrm{G}-\mathrm{C} 18: 1}=C_{\mathrm{Gelatin}}=2.5 \mathrm{mg} \cdot \mathrm{mL}^{-1}\right)$.

The data collected so far show that G-C18:1 interacts with all polyelectrolytes tested in this work and that its micelle-to-vesicle phase transition drives the Co-to- $M L W V$ transition. As one could reasonably expect, the strong electrostatic interactions between the positively- 
charged PEC and negatively-charged G-C18:1 drives the PEC formation across the entire $\mathrm{pH}$ range. To test the solidity of the PESCs synthesis using G-C18:1 and polycations, we employ gelatin as a possible alternative polyelectrolyte and which could be interesting to prepare biobased PESCs. We use a commercial (Aldrich) source of gelatin type A, a natural protein of isoelectric point between 7.0-9.0, below which the charge becomes positive. Figure 5 shows pH-resolved in situ contour plots of gelatin and [G-C18:1 + gelatin] samples. The control gelatin sample in Figure 5a shows no specific contribution across the entire $\mathrm{pH}$ range between $0.1<q / \AA^{-1}<0.4$. Interestingly, the [G-C18:1 + gelatin] sample presented in Figure 5b does not show any signal either in the same $\mathrm{pH}$ and $q$ range, except for the systematic signal of the lamellar, $L$, phase of G-C18:1 below $\mathrm{pH} 4{ }^{40,41}$

Despite an expected positive charge density of gelatin, the in situ SAXS experiment shows no sign of the Co phase above $\mathrm{pH} 7$, indicating that the charge density is probably too low to interact with negatively-charged G-C18:1 micelles. Although somewhat unexpected because interactions with negatively-charged sodium dodecyl sulfate micelles across a wide compositional and $\mathrm{pH}$ range were reported in other studies, ${ }^{66}$ this result is not a surprise. What it is more interesting from a mechanistic point of view is the lack of the $M L W V$ phase below $\mathrm{pH}$ 7. Given its isoelectric point, type A gelatin is positively charged below $\mathrm{pH} 7$ and it is then expected to interact with G-C18:1 negative membranes.

In this work we have used a broad set of polyelectrolytes, of which the different chemical nature let us explore various aspects of their interactions with $\mathrm{G}-\mathrm{C} 18: 1$. If the nature of the polyelectrolyte (stiffness, charge density, ...) is known to strongly affect the morphology and structure of PESCs, ${ }^{14,31}$ in this work we show that: 1) when the Co and $M L W V$ phases are formed, the structure of the corresponding colloidal structures is very similar, whichever the polyelectrolyte used, even if local phenomena like swelling or long-range order may vary from one polyelectrolyte to another. 2) The $C o$ and $M L W V$ phases are only obtained with polyelectrolytes with a net positive charge, that is polycations. 3) The $M L W V$ phase is always preceded by the Co phase, which seems to be a necessary condition to drive the isostructural and isodimensional $C o$-to- $M L W V$ transition. This phenomenon does not occur when gelatin is employed and where the $M L W V$ phase is not observed. On the contrary, the $M L W V$ phase is obtained for the CHL system, despite the fact that we do not have a proof by SAXS of the Co phase. To this regards, we must outline that the SAXS signal for the [G-C18:1 + CHL] system at basic $\mathrm{pH}$ is dominated by the precipitated CHL phase, which we think to be in major amount but not the only phase. Cryo-TEM shows the presence of an unknown fraction of complex coacervates, which we believe to be source of the $M L W V$ phase at $\mathrm{pH}$ below 7 . We also believe 
that the higher disorder of the $M L W V$ phase in the [G-C18:1 + CHL] system (broader first order diffraction peak compared to the PLL-derived $M L W V$ in Figure 4e) could be attributed to the smaller fraction of the initial Co phase. In other words, the presence of a less ordered MLWV phase in the CHL system could then the indirect proof that probably a small fraction of the Co phase forms in the CHL system.

\section{Quantitativity and size control}

If the synthesis of PESCs involving vesicles and polyelectrolytes, and eventually forming $M L W V$, has long been addressed in the literature, ${ }^{36,67,68}$ very few studies, if none, address the issue of quantitativity in relationship to the mechanism of formation. In particular, the synthesis of $M L W V$ from a continuous isostructural phase transition from a coacervate phase has not been addressed before, because $M L W V$ are generally obtained by mixing vesicles and polyelectrolytes in solution. ${ }^{18,28,31-34,36}$ If some authors state that the formation of $M L W V$ is driven by the lipid:polyelectrolyte ratio, other authors show that a mix of agglutinated vesicles and $M L W V$ are actually obtained. ${ }^{37,38}$ Other procedures could probably be followed to increase this control when working with pre-formed vesicles, such as the insertion of the polymer into the hydrophobic vesicle bilayer, which was reported in the case of polycations bearing pendant hydrophobic groups. ${ }^{36,69}$ However, it was found that such interaction could be accompanied by lateral lipid segregation, highly accelerated transmembrane migration of lipid molecules (polycation-induced flip-flop), incorporation of adsorbed polycations into vesicular membrane as well as aggregation and disruption of vesicles. ${ }^{69}$

To evaluate the amount of $M L W V$ with respect to agglutinated vesicles, we compare the sample obtained by continuous Co-to- $M L W V$ phase transition with a sample obtained by the more classical approach consisting in mixing G-C18:1 single-wall vesicles and polyelectrolyte, the main one employed in the literature of $M L W V$. If SAXS can prove the presence of a multilamellar structure, it cannot be easily employed to quantify and discriminate between the two structures. For this reason, instead of SAXS, we evaluate the content of $M L W V$ between the two methods of preparation by combining cryo-TEM with optical microscopy using crossed polarizers. If cryo-TEM can differentiate between agglutination and $M L W V$, its high magnification is poorly compatible with good statistics, unless a large number of images are recorded. On the contrary, optical microscopy using cross polarizers is the ideal technique to differentiate, on the hundreds of micron scale, between $M L W V$ and agglutinated vesicles: multilamellar structures (but not single-wall vesicles) show a characteristic maltese cross 
pattern $^{70}$ under crossed polarizers, found both in concentric lamellar emulsions ${ }^{71}$ and in spherical lamellar structures. ${ }^{72}$

Cryo-TEM of a samples obtained from a Co-to- $M L W V$ phase transition was shown in Figure 3 and, as already commented above, they show a massive presence of vesicular structures having multilamellar walls, as also confirmed by the corresponding SAXS data presented in Figure 4. Figure 6 shows two representative microscopy images of a typical sample prepared with the same approach; images are collected under white $(\mathrm{a}, \mathrm{d})$ and polarized light with polarizers at $0^{\circ}-90^{\circ}(\mathrm{b}, \mathrm{e})$ and $45^{\circ}-135^{\circ}(\mathrm{c}, \mathrm{f})$. The system is characterized by a large number of vesicles highly heterogeneous in size but all below $\sim 10 \mu \mathrm{m}$. Under polarized light and crossed polarizers the entire material displays a typical maltese cross colocalized with each vesicle. Despite the aggregation of the vesicles, also observed with cryo-TEM, maltese crosses are welldefined and nicely separated and each identifying single multilamellar wall vesicles. The entire material displays such a characteristic birefringency, strongly suggesting a quantitative presence of $M L W V$.
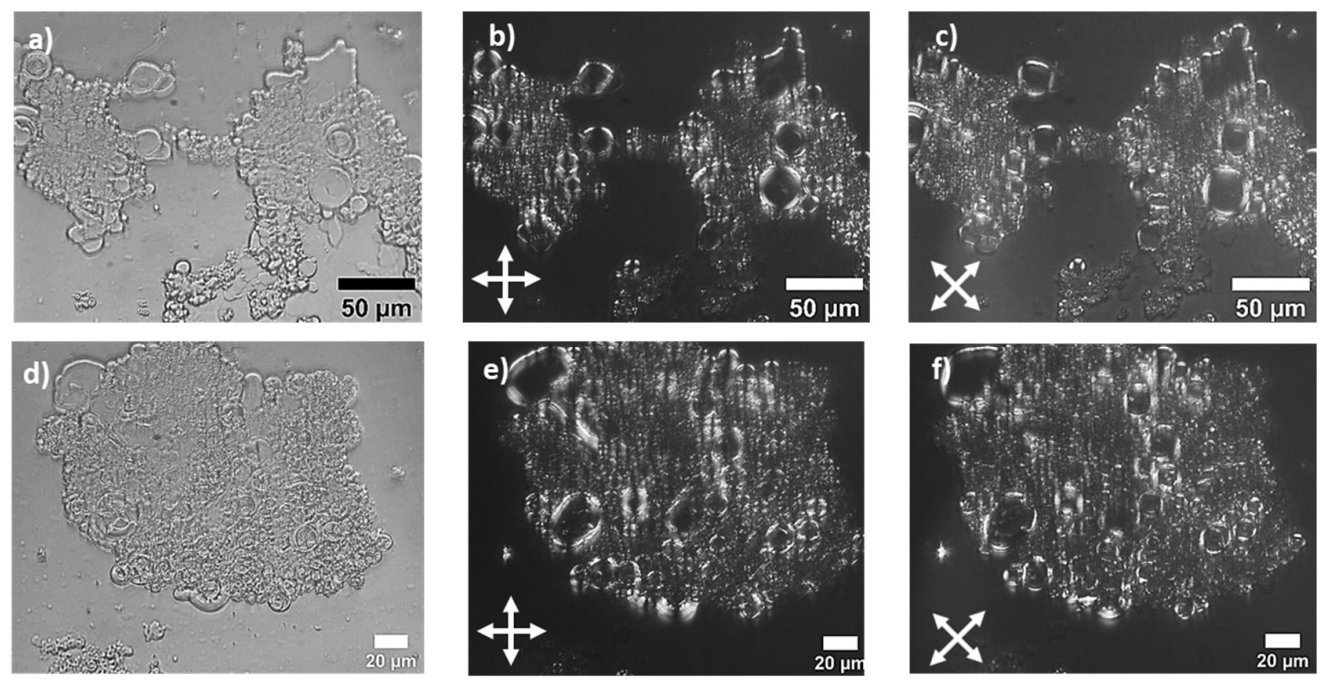

Figure 6 - Optical microscopy images recorded on a $[\mathrm{G}-\mathrm{C} 18: 1+\mathrm{PLL}]$ solution $\left(C_{\mathrm{G}-\mathrm{C18:1}}=C_{\mathrm{PLL}}=2.5 \mathrm{mg} \cdot \mathrm{mL}^{-}\right.$ $\left.{ }^{1}\right)$ at pH 3.9 obtained from a $C o$-to- $M L W V$ phase transition. a,d) white light and polarized light with cross polarizers set at $b, e) 0-90^{\circ}$ and $\left.c, f\right) 45-135^{\circ}$.

The experiment consisting in mixing acidic solutions ( $\mathrm{pH} 3.8$ ) of pre-formed G-C18:1 single-wall vesicles and PLL is shown in Figure 7. A preliminary investigation by optical microscopy results in a different behavior and distribution of signal with respect to the sample obtained through the Co-to- $M L W V$ phase transition. Figure 7a shows representative images of a sample being constituted of aggregated objects, each of size below $1 \mu \mathrm{m}$, expected for G- 
C18:1 vesicles. ${ }^{42}$ The corresponding images recorded using crossed polarizers (Figure $7 \mathrm{~b}, \mathrm{~d}$ ) show a broad, undefined, birefringency associated to the aggregates with little, if no, content of maltese crosses. The featureless, generalized, birefringency signal suggests that $M L W V$ are either not formed or they form in small amounts, in good agreement with the data presented by others. ${ }^{37,38}$ This assumption is confirmed by cryo-TEM images recorded on the same system and showing a mixture of structures including agglutinated vesicles but also "cabbage-like" and multilamellar structures (Figure 7e-f).

The massive presence of $M L W V$ structures obtained through the phase transition process compare to the mixture of structure obtained from a direct mixing of preformed vesiclespolyelectrolyte solutions confirms the crucial role of the complex coacervates in the formation of $M L W V$ : coacervation seems to be a requirement to the extensive formation of vesicular structures with multilamellar walls. ${ }^{40}$ This is also in agreement with the data obtained from the [G-C18:1 + gelatin] system presented in Figure 5 and prepared using the $\mathrm{pH}$ variation approach. Also in that case, the absence of a complex coacervate phase had as a consequence the absence of the $M L W V$ phase. An additional piece of evidence comes from the CHL system, in which the limited amount of the $C o$ phase generates a more disordered $M L W V$ phase. Combination of the data obtained with gelatin and employing the in situ $\mathrm{pH}$ variation with the data obtained by mixing vesicle and polyelectrolyte solutions at a given $\mathrm{pH}$ demonstrates the importance of the precursor Co phase during the phase change method in order to obtain a massive presence of $M L W V$ structures. 

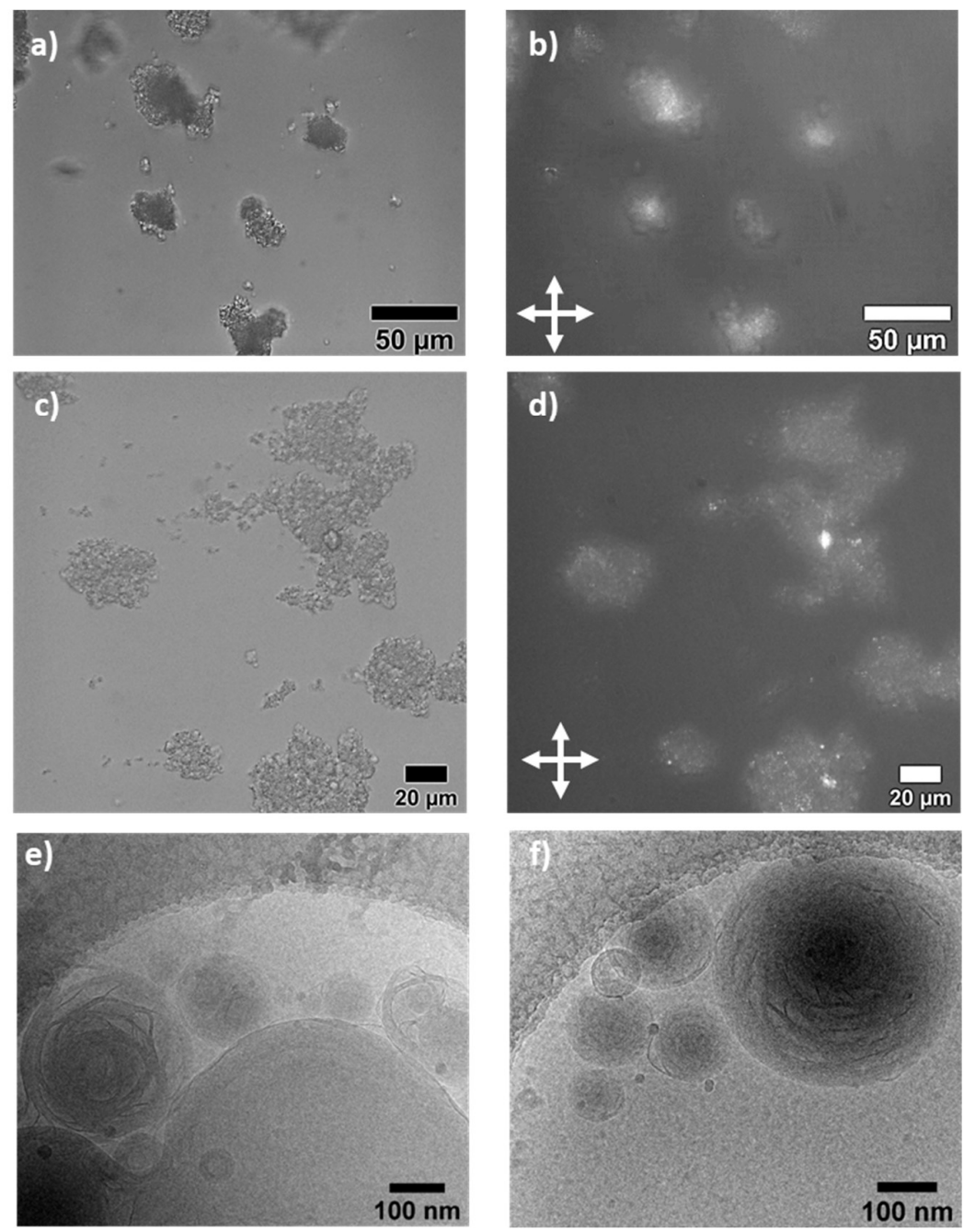

Figure 7 - a-d) Optical microscopy images recorded on a mixture of [G-C18:1] single-wall vesicles and [PLL] solutions ( $\left.C_{\mathrm{G}-\mathrm{C} 18: 1=} C_{\mathrm{PLL}}=2.5 \mathrm{mg} \cdot \mathrm{mL}^{-1}\right)$ both prepared at $\mathrm{pH}=3.8$. a,c) white light and b,d) polarized light with cross polarizers set at $0-90^{\circ}$. Images in e,f) are recorded on the same sample by mean of cryoTEM.

If the $C o$-to- $M L W V$ phase transition is able to quantitatively produce $M L W V$, its main drawback is the poor control over their size distribution, as shown both by TEM and optical microscopy. To improve this point, we employed filtration (Figure 8a-c) and sonication (Figure $8 \mathrm{~d}-\mathrm{f}$ ), these methods being known to efficiently control vesicles size distribution, ${ }^{73}$ but unclear whether or not they have any deleterious impact on the $M L W V$ structure. According to the cryoTEM data in Figure 8a-c, filtration (pore size, $\varphi=450 \mathrm{~nm}$ ) promotes the stabilization of colloidally-stable spherical $M L W V$, of which the diameter seems to be contained between 50 $\mathrm{nm}$ and about $300 \mathrm{~nm}$, in agreement with the filter pore size. Concerning the effect of sonication, Figure 8d-f also shows a large number of spherical, un-aggregated, $M L W V$ colloids, although the diameter appears to be bigger of several hundred nanometers if compared to the filtered 
sample. The cryo-TEM results are confirmed by intensity-filtered DLS experiments, presented in Figure 8g. The as-prepared sample (black curve) shows a $M L W V$ distribution centered at 716 $\mathrm{nm}$, while the filtered sample shows a distribution centered at $460 \mathrm{~nm}$. To better evaluate the impact of sonication, we tested the influence of sonication time and according to DLS data (Figure $8 \mathrm{~g}$ ) we find that at $t=30^{\prime}$ the size distribution is centered at higher diameter values and it is even broader than the as-prepared sample. Applying the same sonication conditions, but over a longer period of time ( $t=1^{\prime}$ or $t=1$ ' 30 ', $)$, it is possible to reduce the $M L W V$ diameter even if the size distribution is broader than the filtration approach, in agreement with the cryoTEM data.

These experiments show that control of the size distribution of $M L W V$ is possible using standard methods employed in liposome science without perturbing the multilamellar wall structure. 

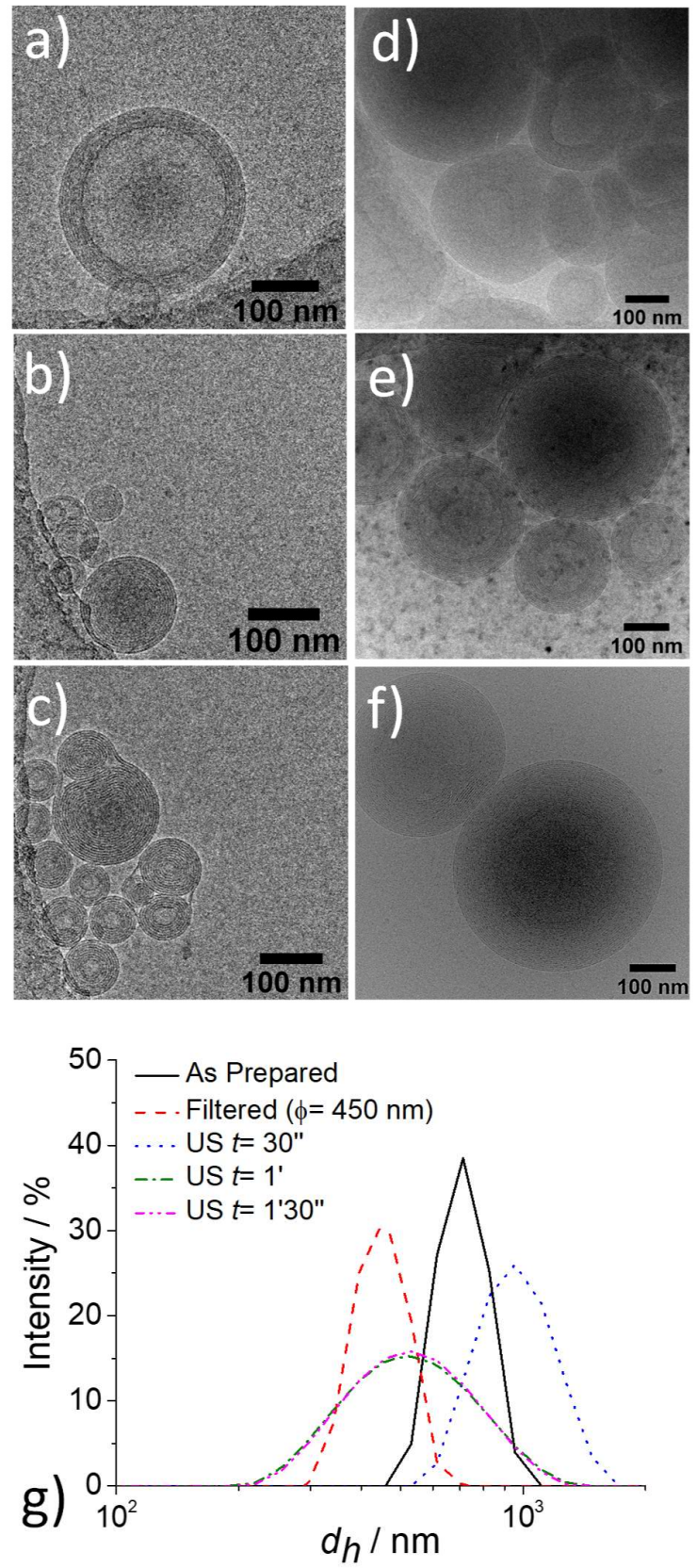

Figure 8 - Cryo-TEM images of a [G-C18:1 + PLL] solution $\left(\mathrm{pH}=5, C_{\mathrm{G}-\mathrm{C} 18: 1}=C_{\mathrm{PLL}}=2.5 \mathrm{mg} \cdot \mathrm{mL}^{-1}\right)$ prepared using the $C o-$-to- $M L W V$ approach and a-c) filtered through a $\varphi=450 \mathrm{~nm}$ pores membrane or d-f) sonicated (ultrasound, US, technical data: $t=1, P=40 \mathrm{~W}$, Ampl.= 40\%, freq.=100\%). g) DLS profiles of the as prepared (black curve), filtered ( $\varphi=450 \mathrm{~nm}$ pores membranes, red curve) and sonicated (US, technical data: $P=40 \mathrm{~W}$, Ampl. $=40 \%$, freq. $=100 \%$, time is given on graph) $M L W V$ samples

\section{Conclusion}


This work addresses the synthesis of multilamellar wall vesicles $(M L W V)$ using a recently-developed method involving a $\mathrm{pH}$-stimulated transition from a complex coacervate phase $(\mathrm{Co})$ instead of a polyelectrolyte-driven vesicle agglutination, classically-employed in the preparation of $M L W V$ polyelectrolyte surfactant complexes (PESCs). We use a combination of a stimuli-responsive microbial glycolipid biosurfactant and a polyamine (either synthetic or natural). The deacetylated acidic C18:1 glucolipid, G-C18:1, undergoes a micelle-to-vesicle phase transition from alkaline to acidic $\mathrm{pH}$. In the alkaline $\mathrm{pH}$ domain, its phase behavior is mainly characterized by negatively-charged micelles. In the presence of a positively-charged polyelectrolyte, G-C18:1 forms a complex coacervate $(\mathrm{Co})$ phase. Upon lowering $\mathrm{pH}$ below the micelle-vesicle boundary, in situ SAXS experiments show a continuous isostructural and isodimensional transition between the $C o$ and $M L W V$ phase. The acidification process reducing the negative charge density, the micellar aggregates embedded in the Co phase undergo a decrease in the local curvature, which drives the transition from spheres to membranes, made of interdigitated G-C18:1 molecules. The membrane has a residual negative charge density, responsible for the strong electrostatic interaction with the polyelectrolyte, crucial to maintain the membranes together. At lower $\mathrm{pH}$, the membrane charge density becomes low and interactions with the polyelectrolyte decrease. This phenomenon promotes intra-chain electrostatic repulsion interactions and eventually encourage the lamellar region to swell. Finally, when the membrane reaches neutrality, polymeric repulsion becomes strong enough to disassemble the lamellae. The polyelectrolyte will most likely be entirely solvated and at sufficiently low $\mathrm{pH}(<3)$ the G-C18:1 precipitates in the form of a lamellar phase, possibly free of the polyelectrolyte, a behavior characteristic of the control lipid solution at the same $\mathrm{pH}$.

We employ four polyelectrolyte, synthetic and natural and with different characteristic of rigidity and charge density (chitosan, poly-L-Lysine, polyethylene imine, polyallylamine); however, the nature of the polyelectrolyte does not seem to be a relevant parameter concerning the fate of the transition, as otherwise found for most PESCs. This may be explained by the strong proximity between the lipid and the polyelectrolyte throughout the isostructural Co-to$M L W V$ transition. If the method described in this work does not allow a tight control over the size distribution of $M L W V$, we also find that the multilamellar wall structure is stable against filtration and sonication, two common methods employed to control the size of vesicles. Last but not least, we show that if we employ the classical approach consisting in mixing pre-formed vesicles with a cationic polyelectrolyte solution at a given $\mathrm{pH}$, we find a much broader structural diversity, including agglutinated single-wall vesicles, multilamellar but also cabbage-like structures, in agreement with previous literature studies. 
All in all, this work establishes the ground for the preparation of a new generation of fully biobased, stimuli-responsive, PESCs, of which the potential fields of applications could span from cosmetics to home-care products.

\section{Acknowledgements}

Diamond synchrotron radiation facility (U. K.) is acknowledged for accessing to the B21 beamline and financial support (proposal $N^{\circ} 23247$ ), as well as Soleil Synchrotron facility for accessing the Swing beamline and financial support (proposal $N^{\circ}$ 20190961). Ghazi Ben Messaoud (DWI-Leibniz Institute for Interactive Materials, Aachen, Germany) is kindly acknowledged for helpful discussions. We thank Dr. S. Roelants, Prof. W. Soetaert and Prof. C. V. Stevens at Gent University for providing us the glycolipid.

\section{Supporting Information: Figure S1}

\section{References}

(1) Morán, M. C.; Miguel, M. G.; Lindman, B. Surfactant-DNA Gel Particles: Formation and Release Characteristics. Biomacromolecules 2007, 8, 3886-3892.

(2) Dobrynin, A. V.; Rubinstein, M. Theory of Polyelectrolytes in Solutions and at Surfaces. Prog. Polym. Sci. 2005, 30, 1049-1118.

(3) Wong, G. C. L. Electrostatics of Rigid Polyelectrolytes. Curr. Opin. Colloid Interface Sci. 2006, 11, 310-315.

(4) Boroudjerdi, H.; Kim, Y. W.; Naji, A.; Netz, R. R.; Schlagberger, X.; Serr, A. Statics and Dynamics of Strongly Charged Soft Matter. Phys. Rep. 2005, 416, 129-199.

(5) White, S. R.; Moore, J. S.; Sottos, N. R.; Krull, B. P.; Santa Cruz, W. A.; Gergely, R. C. R. Restoration of Large Damage Volumes in Polymers. Science (80-. ). 2014, 344, $620-623$.

(6) Prausnitz, M. R.; Langer, R. Transdermal Drug Delivery. Nat. Biotechnol. 2008, 26, $1261-1268$.

(7) Drummond, C. J.; Fong, C. Surfactant Self-Assembly Objects as Novel Drug Delivery Vehicles. Curr. Opin. Colloid Interface Sci. 1999, 4, 449-456.

(8) Singh, M.; Briones, M.; Ott, G.; Hagan, D. O. Cationic Microparticles: A Potent Delivery System for DNA Vaccines. PNAS 2000, 97, 811-816. 
(9) Takahashi, A.; Kawaguchi, M. The Structure of Macromolecules Adsorbed on Interfaces. Adv. Polym. Sci. 1982, 46, 1-65.

(10) Müller, M. Polyelectrolyte Complexes in the Dispersed and Solid State II Application Aspects; 2013.

(11) Petzold, G.; Schwarz, S. Polyelectrolyte Complexes in Flocculation Applications. Adv. Polym. Sci. 2014, 256, 25-65.

(12) Kötz, J.; Kosmella, S.; Beitz, T. Self-Assembled Polyelectrolyte Systems. Prog. Polym. Sci. 2001, 26, 1199-1232.

(13) Langevin, D. Complexation of Oppositely Charged Polyelectrolytes and Surfactants in Aqueous Solutions. A Review. Adv. Colloid Interface Sci. 2009, 147-148, 170-177.

(14) Chiappisi, L.; Hoffmann, I.; Gradzielski, M. Complexes of Oppositely Charged Polyelectrolytes and Surfactants - Recent Developments in the Field of Biologically Derived Polyelectrolytes. Soft Matter 2013, 9, 3896-3909.

(15) Piculell, L.; Norrman, J.; Svensson, A. V.; Lynch, I.; Bernardes, J. S.; Loh, W. Ionic Surfactants with Polymeric Counterions. Adv. Colloid Interface Sci. 2009, 147-148, $228-236$.

(16) Piculell, L. Understanding and Exploiting the Phase Behavior of Mixtures of Oppositely Charged Polymers and Surfactants in Water. Langmuir 2013, 29, 10313 10329.

(17) Lindman, B.; Antunes, F.; Aidarova, S.; Miguel, M.; Nylander, T. PolyelectrolyteSurfactant Association-from Fundamentals to Applications. Colloid J. 2014, 76, 585594.

(18) Gradzielski, M.; Hoffmann, I. Polyelectrolyte-Surfactant Complexes (PESCs) Composed of Oppositely Charged Components. Curr. Opin. Colloid Interface Sci. 2018, 35, 124-141.

(19) Kronberg, B.; Holmberg, K.; Lindman, B. Surfactant-Polymer Systems. In Surface Chemistry of Surfactants and Polymers; Kronberg, B., Holmberg, K., Lindman, B., Eds.; John Wiley \& Sons, Ltd., 2014; pp 271-294.

(20) Chiappisi, L.; Prévost, S.; Grillo, I.; Gradzielski, M. From Crab Shells to Smart Systems: Chitosan-Alkylethoxy Carboxylate Complexes. Langmuir 2014, 30, 10615 10616.

(21) Bilalov, A.; Olsson, U.; Lindman, B. Complexation between DNA and Surfactants and Lipids: Phase Behavior and Molecular Organization. Soft Matter 2012, 8, 1102211033. 
(22) Ferreira, G. A.; Loh, W. Liquid Crystalline Nanoparticles Formed by Oppositely Charged Surfactant-Polyelectrolyte Complexes. Curr. Opin. Colloid Interface Sci. 2017, 32, 11-22.

(23) Kizilay, E.; Dinsmore, A. D.; Hoagland, D. A.; Sun, L.; Dubin, P. L. Evolution of Hierarchical Structures in Polyelectrolyte-Micelle Coacervates. Soft Matter 2013, 9 , $7320-7332$.

(24) Kizilay, E.; Kayitmazer, A. B.; Dubin, P. L. Complexation and Coacervation of Polyelectrolytes with Oppositely Charged Colloids. Adv. Colloid Interface Sci. 2011, 167, 24-37.

(25) Duarte, S.; Faneca, H.; Pedroso De Lima, M. C. Non-Covalent Association of Folate to Lipoplexes: A Promising Strategy to Improve Gene Delivery in the Presence of Serum. J. Control. Release 2011, 149, 264-272.

(26) Dias, R. S.; Dawson, K.; Miguel, M. G. Interaction of DNA with Surfactants in Solution. In DNA Interactions with Polymers and Surfactants; Dias, R., Lindman, B., Eds.; Hoboken, New Jersey, 2008; pp 89-117.

(27) Chen, Y.; Zhu, Q.; Tian, Y.; Tang, W.; Pana, F.; Rulin Xiong, Y.; Yuan, Y.; Hu, A.; 5. Supramolecular Aggregates from Polyacrylates and Gd(III)-Containing Cationic Surfactant as High-Relaxivity MRI Contrast Agents. Polym. Chem. 2015, 6, 15211526.

(28) Zinchenko, A. A.; Pyshkina, O. A.; Lezov, A. V.; Sergeyev, V. G.; Yoshikawa, K. Single DNA Molecules: Compaction and Decompaction. In DNA Interactions with Polymers and Surfactants; Dias, R., Lindman, B., Eds.; John Wiley \& Sons, Inc.: Hoboken, New Jersey, 2008; pp 59-88.

(29) Rafael, D.; Andrade, F.; Arranja, A.; Luís, S.; Videira, M. Lipoplexes and Polyplexes: Gene Therapy. Encycl. Biomed. Polym. Polym. Biomater. 2015, No. August 2016, $4335-4347$.

(30) Golan, S.; Talmon, Y. Nanostructure of Complexes between Cationic Lipids and an Oppositely Charged Polyelectrolyte. Langmuir 2012, 28, 1668-1672.

(31) Ram-On, M.; Cohen, Y.; Talmon, Y. Effect of Polyelectrolyte Stiffness and Solution $\mathrm{PH}$ on the Nanostructure of Complexes Formed by Cationic Amphiphiles and Negatively Charged Polyelectrolytes. J. Phys. Chem. B 2016, 120, 5907-5915.

(32) Weisman, S.; Hirsch-Lerner, D.; Barenholz, Y.; Talmon, Y. Nanostructure of Cationic Lipid-Oligonucleotide Complexes. Biophys. J. 2004, 87, 609-614.

Huebner, S.; Battersby, B. J.; Grimm, R.; Cevc, G. Lipid-DNA Complex Formation: 
Reorganization and Rupture of Lipid Vesicles in the Presence of DNA as Observed by Cryoelectron Microscopy. Biophys. J. 1999, 76, 3158-3166.

Chiappisi, L.; Gradzielski, M. Co-Assembly in Chitosan-Surfactant Mixtures: Thermodynamics, Structures, Interfacial Properties and Applications. Adv. Colloid Interface Sci. 2015, 220, 92-107.

(35) Marques, E. F.; Regev, O.; Khan, A.; Miguel, M. D. G.; Lindman, B. Interactions between Catanionic Vesicles and Oppositely Charged Polyelectrolytes - Phase Behavior and Phase Structure. Macromolecules 1999, 32, 6626-6637.

(36) Antunes, F. E.; Marques, E. F.; Miguel, M. G.; Lindman, B. Polymer-Vesicle Association. Adv. Colloid Interface Sci. 2009, 147-148, 18-35.

(37) De Souza, T. P.; Bossa, G. V.; Stano, P.; Steiniger, F.; May, S.; Luisi, P. L.; Fahr, A. Vesicle Aggregates as a Model for Primitive Cellular Assemblies. Phys. Chem. Chem. Phys. 2017, 19, 20082-20092.

(38) Gasperini, A. A. M.; Puentes-Martinez, X. E.; Balbino, T. A.; De Paula Rigoletto, T.; De Sá Cavalcanti Corrêa, G.; Cassago, A.; Portugal, R. V.; De La Torre, L. G.;

Cavalcanti, L. P. Association between Cationic Liposomes and Low Molecular Weight Hyaluronic Acid. Langmuir 2015, 31, 3308-3317.

(39) Chiappisi, L.; David Leach, S.; Gradzielski, M. Precipitating Polyelectrolyte-Surfactant Systems by Admixing a Nonionic Surfactant-a Case of Cononsurfactancy. Soft Matter 2017, 13, 4988-4996.

(40) Seyrig, C.; Griel, P. Le; Cowieson, N.; Perez, J.; Baccile, N. Stimuli-Induced NonEquilibrium Phase Transitions in Polyelectrolyte-Surfactant Complex Coacervates. ChemRxiv 2020, 10.26434/chemrxiv.12058872.

(41) Baccile, N.; Cuvier, A.-S.; Prévost, S.; Stevens, C. V.; Delbeke, E.; Berton, J.; Soetaert, W.; Van Bogaert, I. N. A.; Roelants, S. Self-Assembly Mechanism of PH-Responsive Glycolipids: Micelles, Fibers, Vesicles, and Bilayers. Langmuir 2016, 32, 1088110894.

(42) Baccile, N.; Selmane, M.; Le Griel, P.; Prévost, S.; Perez, J.; Stevens, C. V.; Delbeke, E.; Zibek, S.; Guenther, M.; Soetaert, W.; et al. PH-Driven Self-Assembly of Acidic Microbial Glycolipids. Langmuir 2016.

(43) Cuvier, A. S.; Berton, J.; Stevens, C. V.; Fadda, G. C.; Babonneau, F.; Van Bogaert, I. N. A.; Soetaert, W.; Pehau-Arnaudet, G.; Baccile, N. PH-Triggered Formation of Nanoribbons from Yeast-Derived Glycolipid Biosurfactants. Soft Matter 2014, 10, 3950-3959. 
(44) Liu, W.; Sun, S.; Cao, Z.; Zhang, X.; Yao, K.; Lu, W. W.; Luk, K. D. K. An Investigation on the Physicochemical Properties of Chitosan/DNA Polyelectrolyte Complexes. Biomaterials 2005, 26, 2705-2711.

(45) Lewis, S. R.; Datta, S.; Gui, M.; Coker, E. L.; Huggins, F. E.; Daunert, S.; Bachas, L.; Bhattacharyya, D. Reactive Nanostructured Membranes for Water Purification. Proc. Natl. Acad. Sci. U. S. A. 2011, 108, 8577-8582.

(46) Mady, M. M.; Mohammed, W. A.; El-Guendy, N. M.; Elsayed, A. A. Effect of Polymer Molecular Weight on the Dna/Pei Polyplexes Properties. J. Biophys 2011, 21, $151-165$.

(47) Hayward, D. W.; Chiappisi, L.; Teo, J. H.; Prévost, S.; Schweins, R.; Gradzielski, M. Neutralisation Rate Controls the Self-Assembly of PH-Sensitive Surfactants. Soft Matter 2019, 15, 8611-8620.

(48) Ben Messaoud, G.; Le Griel, P.; Hermida-Merino, D.; Roelants, S. L. K. W.; Soetaert, W.; Stevens, C. V.; Baccile, N. PH-Controlled Self-Assembled Fibrillar Network (SAFiN) Hydrogels: Evidence of a Kinetic Control of the Mechanical Properties. Chem. Mater. 2019, 31, 4817-4830.

(49) Schindelin, J.; Arganda-Carreras, I.; Frise, E.; Kaynig, V.; Longair, M.; Pietzsch, T.; Preibisch, S.; Rueden, C.; Saalfeld, S.; Schmid, B.; et al. Fiji: An Open-Source Platform for Biological-Image Analysis. Nat. Methods 2012, 9, 676-682.

(50) Ben Messaoud, G.; Promeneur, L.; Brennich, M.; Roelants, S. L. K. W.; Le Griel, P.; Baccile, N. Complex Coacervation of Natural Sophorolipid Bolaamphiphile Micelles with Cationic Polyelectrolytes. Green Chem. 2018, 20, 3371-3385.

(51) Swanson-Vethamuthu, M.; Dubin, P. L.; Almgren, M.; Yingjie, L. Cryo-TEM of Polyelectrolyte - Micelle Complexes. J. Colloid Interface Sci. 1997, 186, 414-419.

(52) Koga, S.; Williams, D. S.; Perriman, A. W.; Mann, S. Peptide-Nucleotide Microdroplets as a Step towards a Membrane-Free Protocell Model. Nat. Chem. 2011, 3, 720-724.

(53) Kayitmazer, A. B.; Strand, S. P.; Tribet, C.; Jaeger, W.; Dubin, P. L. Effect of Polyelectrolyte Structure on Protein - Polyelectrolyte Coacervates: Coacervates of Bovine Serum Albumin with Poly(Diallyldimethylammonium Chloride) versus Chitosan. Biomacromolecules 2007, 8, 3568-3577.

(54) Tanford, C. The Hydrophobic Effect: Formation of Micelles and Biological Membranes; Wiley-Interscience, 1973.

(55) Schiessel, H.; Aranda-Espinosa, H. Electrostatically Induced Undulations of Lamellar 
DNA-Lipid Complexes. Eur. Phys. J. E 2001, 5, 499-506.

Cuvier, A. S.; Babonneau, F.; Berton, J.; Stevens, C. V.; Fadda, G. C.; PéhauArnaudet, G.; Le Griel, P.; Prévost, S.; Perez, J.; Baccile, N. Nanoscale Platelet Formation by Monounsaturated and Saturated Sophorolipids under Basic PH Conditions. Chem. - A Eur. J. 2015, 21, 19265-19277.

(57) Teixeira, J. Small-Angle Scattering by Fractal Systems. J. Appl. Crystallogr. 1988, 21, $781-785$.

(58) Rinaudo, M. Chitin and Chitosan: Properties and Applications. Prog. Polym. Sci. 2006, $31,603-632$.

(59) Sjöström, L.; Åkesson, T.; Jönsson, B. Interaction and Conformation of Polyelectrolyte Chains Adsorbed on Neutral Surfaces. J. Chem. Phys. 1993, 99, 4739-4747.

(60) Fleer, G. J.; Stuart, M. A. C.; Scheutjens, J. M. H. M.; Cosgrove, T.; Vincent, B. Polymers at Interfaces, 1998th ed.; Springer-Science, 1998.

(61) Gerelli, Y.; Di Bari, M. T.; Deriu, A.; Clemens, D.; Almásy, L. Lipid Multilayered Particles: The Role of Chitosan on Structure and Morphology. Soft Matter 2010, 6, $2533-2538$.

(62) Kogej, K.; Evmenenko, G.; Theunissen, E.; Berghmans, H.; Reynaers, H. Investigation of Structures in Polyelectrolyte/Surfactant Complexes by X-Ray Scattering. Langmuir 2001, 17, 3175-3184.

(63) Wang, Y.; Kimura, K.; Dubin, P. L.; Jaeger, W. Polyelectrolyte-Micelle Coacervation: Effects of Micelle Surface Charge Density, Polymer Molecular Weight, and Polymer/Surfactant Ratio. Macromolecules 2000, 33, 3324-3331.

(64) Tarahovsky, Y. S.; Koynova, R.; MacDonald, R. C. DNA Release from Lipoplexes by Anionic Lipids: Correlation with Lipid Mesomorphism, Interfacial Curvature, and Membrane Fusion. Biophys. J. 2004, 87, 1054-1064.

(65) Zhou, S.; Chu, B. Assembled Materials: Polyelectrolyte-Surfactant Complexes. Adv. Mater. 2000, 12, 545-556.

(66) Greener, J.; Contestable, B. A.; Bale, M. D. Interaction of Anionic Surfactants with Gelatin: Viscosity Effects. Macromolecules 1987, 20, 2490-2498.

(67) Bordi, F.; Cametti, C.; Diociaiuti, M.; Gaudino, D.; Gili, T.; Sennato, S. Complexation of Anionic Polyelectrolytes with Cationic Liposomes: Evidence of Reentrant Condensation and Lipoplex Formation. Langmuir 2004, 20, 5214-5222.

(68) Rozenfeld, J. H. K.; Duarte, E. L.; Barbosa, L. R. S.; Lamy, M. T. The Effect of an Oligonucleotide on the Structure of Cationic DODAB Vesicles. Phys. Chem. Chem. 
Phys. 2015, 17, 7498-7506.

(69) Yaroslavov, A.; Kabanov, V. What Happens to Negatively Charged Vesicles upon Interacting with Polycation Species? Am. Chem. Soc. Polym. Prepr. Div. Polym. Chem. 2000, 41, 1613.

(70) Regev, O. Various Bilayer Organizations in a Single-Tail Nonionic Surfactant: Unilamellar Vesicles, Multilamellar Vesicles, and Flat-Stacked Lamellae. Langmuir 1999, 15, 4357-4364.

(71) Park, B. D.; Youm, J. K.; Jeong, S. K.; Choi, E. H.; Ahn, S. K.; Lee, S. H. The Characterization of Molecular Organization of Multilamellar Emulsions Containing Pseudoceramide and Type III Synthetic Ceramide. J. Invest. Dermatol. 2003, 121, 794 801.

(72) Harris, J. K.; Rose, G. D.; Bruening, M. L. Spontaneous Generation of Multilamellar Vesicles from Ethylene Oxide/Butylene Oxide Diblock Copolymers. Langmuir 2002, $18,5337-5342$.

(73) Pereira-Lachataignerais, J.; Pons, R.; Panizza, P.; Courbin, L.; Rouch, J.; López, O. Study and Formation of Vesicle Systems with Low Polydispersity Index by Ultrasound Method. Chem. Phys. Lipids 2006, 140, 88-97. 


\section{Supplementary information}

\section{Synthesis of multilamellar walls vesicles $(M L W V)$ polyelectrolyte surfactant complexes (PESCs) from pH-stimulated phase transition using microbial biosurfactants}

\section{Chloé Seyriga ${ }^{\mathrm{a}}$ Patrick Le Griel ${ }^{\mathrm{a}}$, N. Cowieson, ${ }^{\mathrm{b}}$ J. Perez, ${ }^{\mathrm{c}}$ Niki Baccile ${ }^{\mathrm{a}}$}

a Sorbonne Université, Centre National de la Recherche Scientifique, Laboratoire de Chimie de la Matière Condensée de Paris, LCMCP, F-75005 Paris, France

${ }^{\mathrm{b}}$ Diamond Light Source Ltd, Diamond House, Harwell Science \& Innovation Campus, Didcot, Oxfordshire, OX11 0DE

c Synchrotron SOLEIL, L'Orme des Merisiers Saint-Aubin, BP 4891192 Gif-sur-Yvette Cedex

Content : Figure S1 

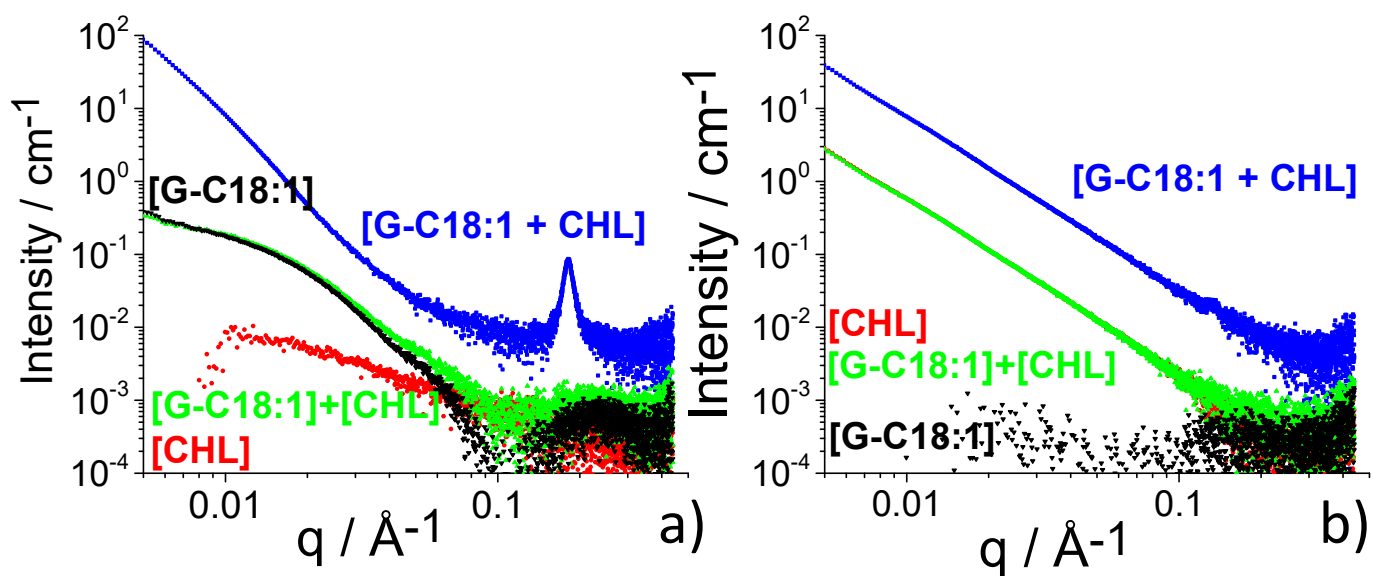

Figure S 1 - SAXS profiles of [G-C18:1] (black) and [CHL] (red) control and [G-C18:1 + CHL] (blue) solutions $\left(C_{\mathrm{G}-\mathrm{C} 18: 1}=2.5 \mathrm{mgmL}^{-1}, C_{\mathrm{CHL}}=1 \mathrm{mg}^{-1} \mathrm{~mL}^{-1}\right)$ at a) $\mathrm{pH}=4.73$ and b) $\mathrm{pH}=8.81$. The green [G-C18:1] + [CHL] profiles correspond to the arithmetic sum of [G-C18:1] + [CHL] individual SAXS profiles. 